\title{
Enabling biomarker discovery in Parkinson's disease using multiomics: challenges, promise and the future
}

\author{
Michael A Kiebish*,1 \& Niven R Narain ${ }^{1}$ \\ ${ }^{1}$ BERG LLC, Precision Medicine Division, Framingham, MA 01701, USA \\ *Author for correspondence: Michael.Kiebish@berghealth.com
}

\begin{abstract}
"biomarker panels to identify prodromal patient populations as well as to stage the disease provide the essential solution that works concomitantly with novel therapeutic development strategies that are under investigation."
\end{abstract}

First draft submitted: 4 September 2018; Accepted for publication: 3 October 2018; Published online: 11 November 2018

Neurodegeneration and neurological disease(s) pose a daunting health economic burden for much of the developed world and in some cases developing nations. Newly discovered genetic risk factors and/or increasing environmental insults are contributing to a surge in disease incidence. Currently, there is a dearth of diagnostic tools available to predict, diagnose or mitigate disease risk or progression, leading to a challenging dilemma within the healthcare management system. Neurodegenerative diseases, such as Alzheimer's or Parkinson's disease strike later in life, where the predominant cost burden falls on governments for treatment or intervention. Alzheimer's disease poses a massive impending challenge, since understanding of disease etiology is still evolving and targeted therapies are still in development with historically suboptimal effects on halting disease progression. However, the biological and physiological deconvolution of Parkinson's disease is better understood with effective therapies that exist. The key for neurodegenerative diseases is identifying patients at risk prior to symptoms or disease onset to delay progression and cost associated of the disease. Thus, biomarkers and diagnostics play a pivotal role in the future treatment paradigm.

Parkinson's disease affects over 10 million patients worldwide, which will increase significantly over the coming decades. In the USA, there are over 1 million cases of Parkinson's disease, resulting in a direct and indirect cost of over $\$ 25$ billion dollars annually to the healthcare system and beyond [1,2]. Unfortunately, only 10-15\% of Parkinson's disease cases can be attributed to genetic factors, resulting in significant portion of individuals with unknown etiology likely exposed to undetermined environmental risk factors. Moreover, given that a large portion of idiopathic Parkinson's disease patients comprise overall incidence, discovering population biomarkers of nongenetic origin that can be used globally becomes essential. Symptoms of Parkinson's disease present upon loss or dysfunction of $60-80 \%$ of the dopaminergic neurons within the substatia nigra [3]. Unfortunately, diagnosis occurs within the clinical phase where oxidative stress has already increased in the brain, nonmotor symptoms are affected and motor symptoms are observable. The predominant goal of biomarker discovery within Parkinson's disease is to identify patients at risk within the prodromal phase of the disease prior to symptoms being clinically detected, thereby preventing an additional neuronal loss leading to exacerbation of disease symptoms [4]. This would allow for more prophylactic intervention of the disease for at-risk patients.

The tools of biomarker discovery have advanced beyond the genomic blueprint, especially for diseases such as Parkinson's disease, which usually occur later in life. Maturing technologies such as clinical proteomics, metabolomics and lipidomics driven by mass spectrometry have been established over the years providing robust quantitative measurements that allow for biomarker discovery, which now are held to strict quantitative guidelines to ensure reproducibility of data. With the advent of high-density quantitative information being generated for biomarker discovery, the advancement of complementary informatics tools derived from machine learning, artificial intelligence and Bayesian statistics assist with translating biomarkers with high confidence and causality associated with the disease and clinical outcome [5]. This has allowed for the most predictive combinations of 
markers to be determined that will allow for informative as well as a positive impact on the treatment outcomes. However, even with the most advanced informatics as well as quantitative platforms, there are several challenges that exist preventing actionable biomarker discovery using protein, lipid or metabolite-based measurements.

A major challenge with biomarker discovery with neurodegenerative disease is that the patient population is enriched for several comorbidities as well as concomitant medications. Evaluating confirmed Parkinson's disease patients of various Hoehn and Yahr as well as UPDRS staging will be treated with a range of L-Dopa, dopamine agonist, L-Dopa replacement/agonist, MAOB inhibitors, amantadine, anticholinergics, apomorphine and other therapeutic interventions, which will impact proteomic, lipidomic and metabolomic profiles [6]. Finding patients who are treatment naive or even asymptomatic is a significant challenge making validation of biomarkers complicated. For biomarker discovery, the key is to work backward, once informative biomarkers are discovered. Performing an assessment on each analyte for the effect of concomitant medication and comorbidities with staging is key to determine if the expression is tied to the treatment or disease pathophysiology. Importantly, if the biomarker is differentially expressed at the lowest characterized stage of the disease, it may be informative to explore individuals in the prodromal phase of the disease, which is fairly asymptomatic based on the performance of the NPV and PPV values for the marker. Further, if biomarkers for Parkinson's disease align with staging and dose responses for front-line therapies, it allows for even greater utility in delivering the right drug, at the right dose, at the right time, which are hallmark components of precision medicine.

For biomarker panels to be clinically useful, they need to have been validated by an optimally powered clinical study design that addresses a clear unmet need within the healthcare space providing accurate guidance to physicians that will allow for reimbursement based on the validated clinical evidence. After validation in a CLIA laboratory, the biomarker panel can be explored even further to asymptomatic patients, seeking reimbursement for screening. From a market perspective, this is a larger population to explore since a major focus of Parkinson's disease and other neurodegenerative diseases is presymptom diagnoses to allow for prophylactic treatment strategies. For screening, biomarker tests have to be relatively economical and supported by higher PPV and/or NPVs to ensure false positives and negatives are avoided. Hence, there is a clear balance for performance with the economic utility of the test to be successful that will impact disease outcome.

Going beyond the genome, the 'phenome' which is made up of actionable downstream analytes in various tissues and biofluids allowing for the opportunity to explore noninvasive biofluids for biomarker discovery in neurological diseases. Ideally, in any biomarker discovery, it is ideal to evaluate the fluid closest to the tissue of disease origin. Cerebrospinal fluid would be a logical matrix, although not ideal for screening or staging of disease such as Parkinson's disease due to complications of collection and invasiveness. Plasma and/or serum are part of conventional diagnostic screening, thus ideally suited for biomarker discovery, however, OMICS such as proteomics, metabolomics and lipidomics are generally challenged do to the overarching influence of comorbities in characterizing these matrices as well as the fact that it is dissociated from the brain. More noninvasive matrices such as urine, saliva, sweat or intraocular fluid pose attractive avenues due to concentration of environment factors influencing the phenome in urine, as well as physiological signatures of the sympathetic nervous system being altered in saliva, sweat or intraocular fluid. Further, due to the noninvasive collection opportunities of urine, saliva, sweat or intraocular fluid alternative collection procedures such dried paper spotting, which can stabilize diverse analytes will allow for patient-driven collection of samples, if analytes are confirmed to be stable under various perturbations on selected paper [7].

Biomarker discovery in Parkinson's disease is riddled with numerous challenges in using mass spectrometry-based tools due to challenges in demographic, comorbidities and concomitant medications that dramatically impact the utility of a panel of markers, however, there is still tremendous opportunity in this space [8]. In the era of highdensity data collection, informatic tools such as artificial intelligence, machine learning and Bayesian artificial intelligence are essential to illuminate the biomarker signal from the noise, allowing analytes to be identified that impact clinical outcome. Importantly, the primary focus starts with ensuring that the right clinical study design is employed to power and address reimbursable clinical questions that will be supported by patients, physicians and insurance providers. Furthermore, an investigation of noninvasive biofluids that can eventually be collected directly by patients will provide greater utility for screening, which will be driven based on the clinical utility of the panel impacting patient outcomes. With the increase of anxiety, environmental toxins and neurological diseases, Parkinson's disease and other neurological diseases will have a significant increasing impact on the US and other healthcare systems. Taken together, biomarker panels to identify prodromal patient populations as well as to stage the disease provide the essential solution that works concomitantly with novel therapeutic development strategies that 
are under investigation. The aforementioned philosophy and employment of cutting-edge technologies may unravel an in-depth biological network understanding toward dual efforts in biomarker and therapeutic development to serve patients more effectively.

Financial \& competing interests disclosure

The authors have no relevant affiliations or financial involvement with any organization or entity with a financial interest in or financial conflict with the subject matter or materials discussed in the manuscript. This includes employment, consultancies, honoraria, stock ownership or options, expert testimony, grants or patents received or pending, or royalties.

No writing assistance was utilized in the production of this manuscript.

\section{References}

1. Kowal SL, Dall TM, Chakrabarti R, Storm MV, Jain A. The current and projected economic burden of Parkinson's disease in the United States. Mov. Disord. 28, 311-318 (2013).

2. Parkinson's Foundation. Understanding Parkinson's. (2018) www.parkinson.org

3. Kordower JH, Olanow CW, Dodiya HB et al. Disease duration and the integrity of the nigrostriatal system in Parkinson's disease. Brain 136, 2419-2431 (2013).

4. Rees RN, Acharya AP, Schrag A, Noyce AJ. An early diagnosis is not the same as a timely diagnosis of Parkinson's disease. F1000Res doi: 10.12688/f1000research.14528.1 (2018) (Epub ahead of print).

5. Vemulapalli V, Qu J, Garren JM etet al. Nonobvious correlations to disease management unraveled by Bayesian artificial intelligence analyses of CMS data. Artif. Intell. Med. 74, 1-8 (2016).

6. Rizek P, Kumar N, Jog MS. An update on the diagnosis and treatment of Parkinson disease. CMAJ 188, 1157-1165 (2016).

7. Drolet J, Tolstikov V, Williams BA et al. Integrated metabolomics assessment of human dried blood spots and urine strips. Metabolites 7(3), doi: 10.3390/metabo7030035 (2017).

8. Liu Y, Buil A, Collins BC et al. Quantitative variability of 342 plasma proteins in a human twin population. Mol. Syst. Biol. 11(1), 786 (2015) 


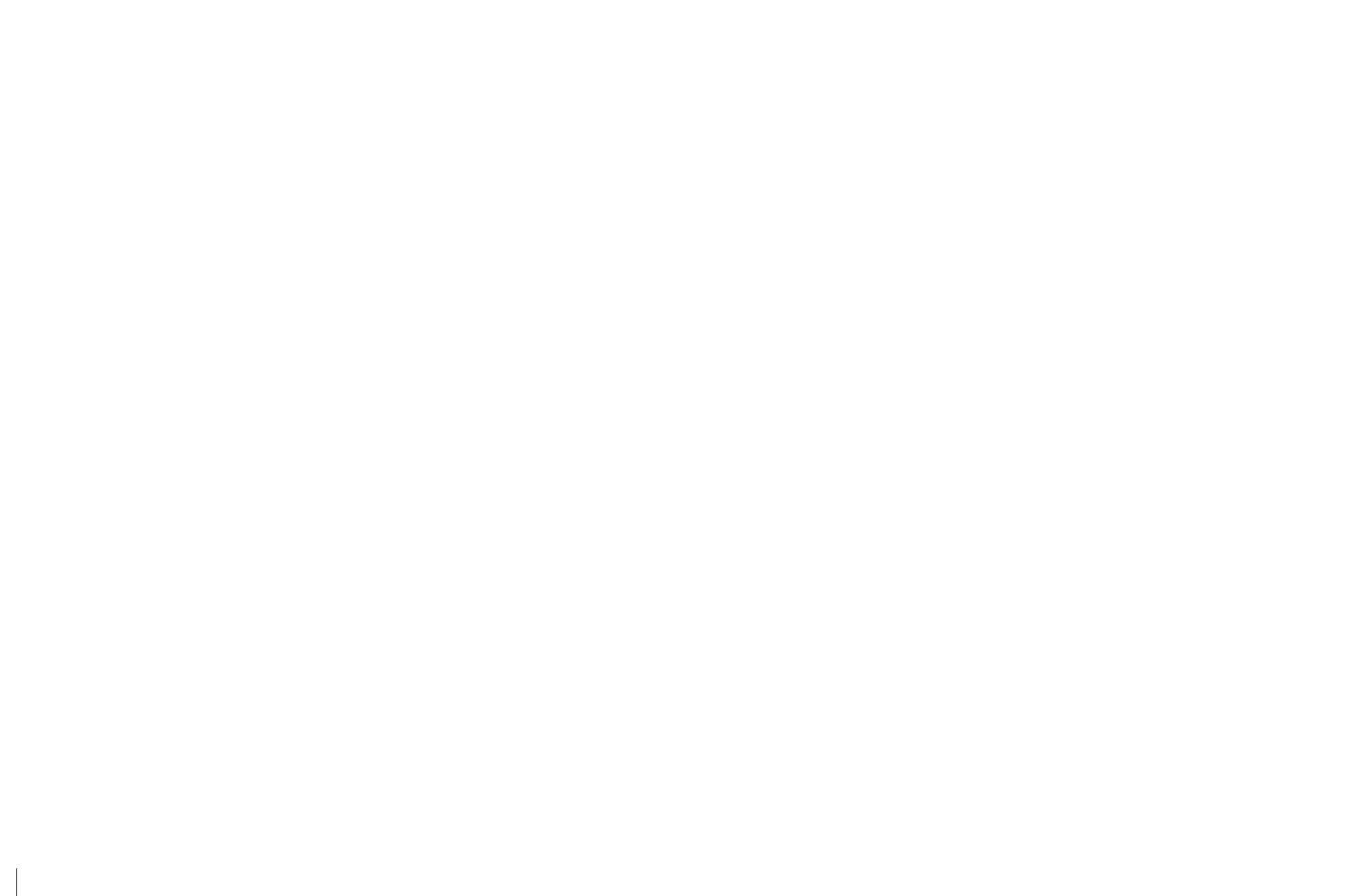

